\title{
Some rigorous relations for partial conductivities in ionic liquids
}

\author{
I. Mryglod 112 , V. Kuporov 11 \\ 1 Institute for Condensed Matter Physics of the National Academy of Sciences of Ukraine, \\ 1 Svientsitskii Str., 79011 Lviv, Ukraine \\ 2 Institute of Applied Mathematics and Fundamental Sciences of Lviv Polytechnic National University, \\ 79013 Lviv, Ukraine
}

Received November 15, 2010

\begin{abstract}
Starting with the rigorous expressions, derived previously for the generalized transport coefficients of a multicomponent fluid, we obtained several exact relations for partial conductivities of ionic charge-asymmetric mixtures. For a simpler case of a charge-symmetric binary mixture such kind of relations was discovered experimentally by Sundheim more than 50 years ago and is known as the "universal golden rule". Some more complicate models, describing in particular the cases of ternary and multi-component mixtures, are considered. The general relation for partial ionic conductivities is derived for a multi-component ionic fluid. It is shown that such relations can be considered in fact as an example of a more general class of rigorous expressions valid for $(k, \omega)$-dependent quantities.
\end{abstract}

Key words: Ionic liquids, transport coefficients, mutual diffusion coefficient, ionic conductivity, molten salt

PACS: $66.10 . \mathrm{cg}, 66.10 . \mathrm{Ed}, 82.45 . \mathrm{Gj}, 47.10 . \mathrm{g}$

\section{Introduction}

About 50 years ago a phenomenological "universal golden rule" for the ratio of partial conductivities of ions in molten salts was proposed by Sundheim [1] from the analysis of experimental data. This rule is expressed in a very simple form

$$
\frac{\sigma_{+}}{\sigma_{-}}=\frac{m_{-}}{m_{+}} .
$$

Recently, there were made several theoretical attempts [2] to derive this relation using the equations of motion, the Langevin equation as well as molecular dynamics studies for the model of binary charge symmetrical molten salts. A few years later using similar approaches such relation was also obtained for pseudo-binary molten salt $\mathrm{KCl}-\mathrm{NaCl}[3]$.

Our goal is to consider this problem in a more general framework. We start with the rigorous relations derived by us previously for generalized transport coefficients of a multi-component fluid [4, 5]. We obtain the "universal golden rule" for $(k, \omega)$-dependent partial conductivities of an ionic charge-asymmetric binary mixture as well as the relations for the partial ionic conductivities in some cases of ternary and four-component ionic liquids.

\section{Theoretical framework}

Let us start with some introductory remarks and consider the general framework that can be used for the description of both kinds of multi-component mixtures, in particular mixtures of neutral particles as well as mixtures containing charged particles. In general case we deal with a $\nu$-component fluid in the volume $V$, containing $N_{\alpha}$ particles in the $\alpha$ th species $(\alpha=1,2, \ldots, \nu)$. To derive hydrodynamic equations one has to define the microscopic basic set of the slowest (hydrodynamic) variables [5], which for a multi-component mixture may be introduced as follows 
$\hat{P}_{\mathbf{k}}^{\text {hyd }}=\left\{\hat{\mathcal{N}}_{\mathbf{k}}, \hat{\mathbf{J}}_{\mathbf{k}}, \hat{E}_{\mathbf{k}}\right\}$, where $\hat{\mathcal{N}}_{\mathbf{k}}=\left\{\hat{N}_{\mathbf{k}, \alpha}\right\}$ is a column-vector with the components

$$
\hat{N}_{\mathbf{k}, \alpha}=\sum_{i=1}^{N_{\alpha}} \exp \left\{\mathbf{i k R}_{i}^{\alpha}\right\}
$$

being the number density of particles in the $\alpha$-th species; $\hat{\mathbf{J}}_{\mathbf{k}}$ is the density of the total current,

$$
\hat{\mathbf{J}}_{\mathbf{k}}=\sum_{\alpha} \hat{\mathbf{J}}_{\mathbf{k}, \alpha}, \quad \hat{\mathbf{J}}_{\mathbf{k}, \alpha}=\sum_{i=1}^{N_{\alpha}} \mathbf{p}_{i}^{\alpha} \exp \left\{\mathrm{i} \mathbf{k} \mathbf{R}_{i}^{\alpha}\right\}
$$

with $\hat{\mathbf{J}}_{\mathbf{k}, \alpha}$ being the current densities of particles in the $\alpha$-th species, and

$$
\hat{E}_{\mathbf{k}}=\sum_{\alpha} \hat{E}_{\mathbf{k}, \alpha}=\sum_{\alpha} \sum_{i=1}^{N_{\alpha}} e_{i}^{\alpha} \exp \left\{\mathrm{ik} \mathbf{R}_{i}^{\alpha}\right\}
$$

is the total energy density, where the one-particle energy $e_{j}^{\alpha}$ can be expressed via the sum of kinetic energy and potential energy of pair interactions:

$$
e_{j}^{\alpha}=\frac{\left[\mathbf{p}_{j}^{\alpha}\right]^{2}}{2 m_{\alpha}}+\sum_{l \neq j, \beta} V_{j l}^{\beta \alpha} .
$$

The set of dynamic variables $\hat{P}_{\mathbf{k}}^{\text {hyd }}=\left\{\hat{\mathcal{N}}_{\mathbf{k}}, \hat{\mathbf{J}}_{\mathbf{k}}, \hat{E}_{\mathbf{k}}\right\}$ includes the densities of all the additive integrals of motion for a mixture. In the case of longitudinal dynamics, as it follows from the symmetrical properties, the scalar densities $\hat{n}_{\mathbf{k}, \alpha}$ and $\hat{E}_{\mathbf{k}}$ interact only with the longitudinal component of $\hat{\mathbf{J}}_{\mathbf{k}}$, namely $\hat{J}_{\mathbf{k}}^{\mathrm{L}}$, that is the projection of $\hat{\mathbf{J}}_{\mathbf{k}}$ onto the direction of wave-vector $\mathbf{k}$. Hence, the total number of longitudinal hydrodynamic variables for $\nu$-component mixture is equal to $\nu+2$, namely $\hat{P}_{\mathbf{k}}^{\mathrm{L}}=\left\{\hat{P}_{\mathbf{k}}^{\iota}\right\}$ with $\iota=1,2, \ldots, \nu+2$.

In practical applications it may be more convenient [4, 5] to use the set of orthogonalized dynamic variables possessing the following properties $\left(\hat{P}_{\mathbf{k}}^{\iota}, \hat{P}_{-\mathbf{k}}^{\kappa}\right)=\delta_{\iota \kappa}\left(\hat{P}_{\mathbf{k}}^{\iota}, \hat{P}_{-\mathbf{k}}^{\kappa}\right)$. Such an orthogonalized set of longitudinal hydrodynamical variables can be defined as follows

$$
\hat{P}_{\mathbf{k}}^{\mathrm{L}}=\left\{\hat{\mathcal{N}}_{\mathbf{k}}, \hat{J}_{\mathbf{k}}^{\mathrm{L}}, \hat{H}_{\mathbf{k}}\right\}
$$

where

$$
\hat{H}_{\mathbf{k}}=\hat{E}_{\mathbf{k}}-\left(\hat{E}_{\mathbf{k}}, \hat{\mathcal{N}}_{\mathbf{k}}^{+}\right)\left(\hat{\mathcal{N}}_{\mathbf{k}}, \hat{\mathcal{N}}_{\mathbf{k}}^{+}\right)^{-1} \hat{\mathcal{N}}_{\mathbf{k}}=\left(1-\mathcal{P}_{\mathcal{N}}\right) \hat{E}_{\mathbf{k}}
$$

is the so-called enthalpy density, the Mori-like projection operator denotes as

$$
\mathcal{P}_{\mathcal{N}} \ldots=\left(\ldots, \mathcal{N}_{\mathbf{k}}^{+}\right)\left(\hat{\mathcal{N}}_{\mathbf{k}}, \hat{\mathcal{N}}_{\mathbf{k}}^{+}\right)^{-1} \hat{\mathcal{N}}=\sum_{\alpha \gamma}\left(\ldots, \hat{N}_{-\mathbf{k}, \alpha}\right)\left(\hat{\mathcal{N}}_{\mathbf{k}}, \hat{\mathcal{N}}_{\mathbf{k}}^{+}\right)_{\alpha \gamma}^{-1} \hat{N}_{\mathbf{k}, \gamma}
$$

and the notation $(\ldots, \ldots)$ is used for the definition of an equilibrium correlation function

$$
(A, B)=\langle(A-\langle A\rangle)(B-\langle B\rangle)\rangle
$$

with $\langle\ldots\rangle$ denoting the equilibrium averaging.

The generalized hydrodynamic fluxes $I_{\mathbf{k}, \iota}^{d}$ can be defined in the standard way:

$$
\mathrm{i} k I_{\mathbf{k}, \iota}^{d}=\left(1-\mathcal{P}_{\mathrm{H}}\right) \mathrm{i} L_{N} \hat{P}_{\mathbf{k}}^{\iota},
$$

where $\mathcal{P}_{\mathrm{H}}$ is the Mori-like projection operator, constructed on the set of all hydrodynamic variables (5), and $\mathrm{i} L_{N}$ is the Liouville operator. Thus, for the number density flux one can easily obtain

$$
\mathrm{i} k I_{\mathbf{k}, \alpha}^{d}=\frac{\mathrm{i} k}{m_{\alpha}}\left(\hat{J}_{\mathbf{k}, \alpha}^{\mathrm{L}}-\frac{m_{\alpha} c_{\alpha}}{\bar{m}} \hat{J}_{\mathbf{k}}^{\mathrm{L}}\right)
$$


where $m_{\alpha}$ is the particle mass in the $\alpha$-th species, $c_{\alpha}=N_{\alpha} / N=n_{\alpha} / n$ denotes concentrations, and $\bar{m}=\sum_{\alpha} c_{\alpha} m_{\alpha}$ is the mean mass per particle.

The generalized $(k, z)$-dependent transport coefficients are defined via the generalized fluxes $I_{\mathbf{k}, \iota}^{d}$ as follows [4, 5]

$$
L_{\iota \kappa}(k, z)=\frac{\beta}{V} \int_{0}^{\infty} \mathrm{d} t \exp \{-z t\}\left(I_{\mathbf{k}, \iota}^{d}, \exp \left\{-\left(1-\mathcal{P}_{\mathrm{H}}\right) \mathrm{i} L_{N} t\right\} I_{-k, \kappa}^{d}\right) .
$$

Note that the expression (9) has the structure of the well-known Green-Kubo formulas [6], but the evolution operator is more complicated and additionally involves the projection operator $\mathcal{P}_{\mathrm{H}}$. However, in the hydrodynamic limit $(k, z) \rightarrow 0$ one gets the expression

$$
L_{\iota \kappa}=\lim _{k, z \rightarrow 0} L_{\iota \kappa}(k, z)=\frac{\beta}{V} \int_{0}^{\infty} \mathrm{d} t\left(I_{\iota}^{d}, \exp \left\{-\mathrm{i} L_{N} t\right\} I_{\kappa}^{d}\right),
$$

that is commonplace in numerous textbooks (e.g. 7]) and has been routinely employed in computer simulations.

In the context of this paper we are mainly interested in the behavior of the generalized mutual diffusion coefficients $D_{\alpha \gamma}(k, z)$ that are simply related to the corresponding transport coefficients $L^{\alpha \gamma}(k, z)$. Namely, one has

$$
L^{\alpha \gamma}(k, z)=n c_{\alpha} c_{\gamma} D_{\alpha \gamma}(k, z) / k_{\mathrm{B}} T,
$$

where $n=N / V$. The explicit frequency dependence in (91) can be found by taking into account that $z=\mathrm{i} \omega+\varepsilon$ and $\varepsilon \rightarrow+0$.

In general for a $\nu$-component mixture the matrix of mutual diffusion coefficients $\mathbf{D}(k, \omega)=$ $\left\|D_{\alpha \gamma}(k, \omega)\right\|$ has $\nu \times \nu$ elements. Due to the symmetry properties $D_{\alpha \gamma}(k, \omega)=D_{\gamma \alpha}(k, \omega)$ this number is reduced to the $\nu(\nu+1) / 2$ independent elements. However, there are still additional $\nu$ explicit relations that follow from the total momentum conversation law. Namely, taking into account that

$$
\sum_{\alpha=1}^{\nu} m_{\alpha} I_{\mathbf{k}, \alpha}^{d}=\sum_{\alpha=1}^{\nu}\left(\hat{J}_{\mathbf{k}, \alpha}^{\mathrm{L}}-\frac{m_{\alpha} c_{\alpha}}{\bar{m}} \hat{J}_{\mathbf{k}}^{\mathrm{L}}\right) \equiv 0
$$

and using the definition (9), the set of new useful relations for generalized transport coefficients, that involve the processes caused by number densities fluctuations, can be easily derived. In particular, one gets

$$
\sum_{\alpha=1}^{\nu} m_{\alpha} c_{\alpha} D_{\alpha \gamma}(k, \omega)=\sum_{\gamma=1}^{\nu} D_{\alpha \gamma}(k, \omega) c_{\gamma} m_{\gamma} \equiv 0 .
$$

Hence, taking into account the relations (13) one can conclude that the total number of independent matrix elements in the matrix $\mathbf{D}(k, \omega)=\left\|D_{\alpha \gamma}(k, \omega)\right\|$ is equal to $\nu(\nu-1) / 2$. In a particular case of binary mixture $(\nu=2)$ we have $\nu(\nu-1) / 2=1$ and

$$
\frac{D_{11}(k, \omega)}{D_{12}(k, \omega)}=-\frac{m_{2}}{m_{1}} \frac{c_{2}}{c_{1}}, \quad \frac{D_{11}(k, \omega)}{D_{22}(k, \omega)}=\frac{m_{2}^{2}}{m_{1}^{2}} \frac{c_{2}^{2}}{c_{1}^{2}} .
$$

These relations directly follow from the identity (13). Being valid for an arbitrary binary mixture, they are of rather general character.

\section{Binary mixture of charged particles}

Let us now consider the case of a binary mixture composed of oppositely charged particles with charges $q_{+}$and $q_{-}$, masses $m_{+}$and $m_{-}$, and densities $n_{+}, n_{-}$. The total electroneutrality condition is satisfied, so that $q_{+} n_{+}+q_{-} n_{-}=0$. 
The electrical conductivity can be calculated by means of the Green-Kubo formula [8, 9]

$$
\sigma=\frac{\beta}{V} \int_{0}^{\infty} \mathrm{d} t\left\langle\mathbf{I}^{q}(t) \mathbf{I}^{q}(0)\right\rangle
$$

where $\mathbf{I}^{q}(t)=\sum_{\alpha} \mathbf{I}_{\alpha}^{q}(t)$ with

$$
\mathbf{I}_{\alpha}^{q}(t)=q_{\alpha} n_{\alpha} \sum_{i=1}^{N_{\alpha}} \mathbf{v}_{i}^{\alpha}
$$

being the partial ionic electrical current $(\alpha=+,-)$. Ionic conductivity is also connected with the mutual diffusion coefficients [9]:

$$
\sigma=\frac{n}{k_{\mathrm{B}} T} \sum_{\alpha, \beta} q_{\alpha} q_{\beta} c_{\alpha} c_{\beta} D_{\alpha \beta}
$$

where mutual diffusion coefficients are defined as follows

$$
D_{\alpha \beta}=\frac{N}{N_{\alpha} N_{\beta}} \sum_{i, j=1}^{N_{\alpha}, N_{\beta}} \int_{0}^{\infty} \mathrm{d} t\left\langle\mathbf{v}_{i}^{\alpha}(t) \mathbf{v}_{j}^{\beta}(0)\right\rangle
$$

with $\alpha, \beta=+,-$. In the center of mass reference frame the expression (16) can be easily derived from (15) with the help of equations (8), (9) and (11). For generalized $(k, \omega)$-dependent ionic conductivity one can use the definition

$$
\sigma(k, \omega)=\frac{n}{k_{\mathrm{B}} T} \sum_{\alpha, \beta} q_{\alpha} q_{\beta} c_{\alpha} c_{\beta} D_{\alpha \beta}(k, \omega) .
$$

It is seen from (17) that the total ionic conductivity can be presented as the sum of partial ionic conductivity $\sigma=\sigma_{+}+\sigma_{-}$, where $\sigma_{\alpha} \sim q_{\alpha} c_{\alpha} \sum_{\beta} q_{\beta} c_{\beta} D_{\alpha \beta}$, so that for the ratio of partial ionic conductivity one gets

$$
\frac{\sigma_{+}}{\sigma_{-}}=\frac{q_{+}^{2} c_{+}^{2} D_{++}+q_{+} c_{+} q_{-} c_{-} D_{+-}}{q_{-}^{2} c_{-}^{2} D_{--}+q_{+} c_{+} q_{-} c_{-} D_{+-}} .
$$

This expression can be significantly simplified if we use the relations that follow from (14), namely: $D_{++} / D_{--}=\left(m_{-}^{2} / m_{+}^{2}\right)\left(c_{-}^{2} / c_{+}^{2}\right), D_{++} / D_{+-}=-\left(m_{-} / m_{+}\right)\left(c_{-} / c_{+}\right), D_{--} / D_{-+}=$ $-\left(m_{+} / m_{-}\right)\left(c_{+} / c_{-}\right), D_{+-}=D_{-+}$. Taking into account the electroneutrality condition $q_{+} c_{+}+$ $q_{-} c_{-}=0$, one gets

$$
\frac{\sigma_{+}(k, \omega)}{\sigma_{-}(k, \omega)}=-\frac{q_{+}}{q_{-}} \frac{m_{-}}{m_{+}} .
$$

In the case of charge-symmetric systems with $q_{-}=-q_{+}$(in particular, for molten salts $\mathrm{NaCl}, \mathrm{KCl}$, $\mathrm{NaF}, \mathrm{KF}, \mathrm{RbBr}$ ) we obtain

$$
\frac{\sigma_{+}(k, \omega)}{\sigma_{-}(k, \omega)}=\frac{m_{-}}{m_{+}} .
$$

In fact, the expression (20) represents the generalized version of the so-called "universal golden rule" (11) valid for binary charge-symmetric ionic liquids with arbitrary values $(k, \omega)$. In a more general form (19) such a relation is derived for a charge-asymmetric binary mixture by means of the rigorous expressions (13) obtained for the generalized mutual diffusion coefficients of a multicomponent fluid. 


\section{Ternary mixtures}

\subsection{Charged particles in solvent}

Let us consider a more complicated model of a ternary mixture that is composed of oppositely charged particles in neutral solvent with the particle charges $q_{+}$and $q_{-}$, the particle masses $m_{+}$, $m_{-}$, and $m_{0}$, and concentrations $c_{+}, c_{-}, c_{0}\left(c_{+}+c_{-}+c_{0}=1\right)$. The total electro-neutrality condition can be written in the form: $q_{+} c_{+}+q_{-} c_{-}=0$.

From the relations (13) one obtains the equations

$$
\begin{aligned}
m_{+} c_{+} D_{++}(k, \omega)+m_{-} c_{-} D_{-+}(k, \omega)+m_{0} c_{0} D_{0+}(k, \omega) & \equiv 0, \\
m_{+} c_{+} D_{+-}(k, \omega)+m_{-} c_{-} D_{--}(k, \omega)+m_{0} c_{0} D_{0-}(k, \omega) & \equiv 0, \\
m_{+} c_{+} D_{+0}(k, \omega)+m_{-} c_{-} D_{-0}(k, \omega)+m_{0} c_{0} D_{00}(k, \omega) & \equiv 0,
\end{aligned}
$$

where $D_{\alpha \beta}=D_{\beta \alpha}$. The electroneutrality condition enables us to express the concentrations of charged particles via $c_{0}$, namely: $c_{+}=\left(1-c_{0}\right) q_{-} /\left(q_{-}-q_{+}\right), c_{-}=\left(1-c_{0}\right) q_{+} /\left(q_{+}-q_{-}\right)$. It is obvious that

$$
\begin{aligned}
q_{+}^{2} c_{+}^{2} & =q_{-}^{2} c_{-}^{2}=\left(1-c_{0}\right)^{2} \frac{q_{+}^{2} q_{-}^{2}}{\left(q_{+}-q_{-}\right)^{2}} \equiv Q^{2}, \\
q_{+} c_{+} q_{-} c_{-} & =-\left(1-c_{0}\right)^{2} \frac{q_{+}^{2} q_{-}^{2}}{\left(q_{+}-q_{-}\right)^{2}} \equiv-Q^{2} .
\end{aligned}
$$

Therefore, one gets $\sigma_{+}=Q^{2}\left(D_{++}-D_{+-}\right)$and $\sigma_{-}=Q^{2}\left(D_{--} D_{+-}\right)$, so that the expression (18) for the ratio of partial ionic conductivities can be rewritten in the form

$$
\frac{\sigma_{+}(k, \omega)}{\sigma_{-}(k, \omega)}=\frac{D_{++}(k, \omega)-D_{+-}(k, \omega)}{D_{--}(k, \omega)-D_{+-}(k, \omega)} .
$$

Combining the first two equations in (21), one can obtain:

$$
m_{+} c_{+}\left(D_{++}-D_{+-}\right)-m_{-} c_{-}\left(D_{--}-D_{+-}\right)+m_{0} c_{0}\left(D_{0+}-D_{0-}\right) \equiv 0
$$

or

$$
m_{+} c_{+} \sigma_{+}-m_{-} c_{-} \sigma_{-}+m_{0} c_{0} \Delta=0,
$$

where the quantity $\Delta \equiv Q^{2}\left(D_{0+}-D_{0-}\right)$ is expressed in terms of the mutual diffusion coefficients for ions in solvent. The relation (24) could be considered as the generalization of "universal golden rule" for solutions of electrolytes. Note that in the limit $c_{0} \rightarrow 0$ the expression (22) can be easily recovered from (22).

\subsection{Pseudo-binary molten salts}

A special class of ternary ionic liquids is formed by the so-called pseudo-binary molten salts, for instance $\mathrm{KCl}-\mathrm{NaCl}$. In this case we deal with a ternary mixture of ions. In particular, for $\mathrm{KCl}-\mathrm{NaCl}$ in the system with the elementary charge $e=1$ the ionic charges are $q_{\mathrm{Na}}=q_{\mathrm{K}}=1$ and $q_{\mathrm{Cl}}=-1$ with the electro-neutrality condition $c_{\mathrm{Na}}+c_{\mathrm{K}}=c_{\mathrm{Cl}}$, where $c_{\mathrm{Na}}+c_{\mathrm{K}}+c_{\mathrm{Cl}}=1$.

For the mutual diffusion coefficients from (13) one has:

$$
\begin{aligned}
& m_{1} c_{1} D_{11}(k, \omega)+m_{2} c_{2} D_{21}(k, \omega)+m_{3} c_{3} D_{31}(k, \omega) \equiv 0, \\
& m_{1} c_{1} D_{12}(k, \omega)+m_{2} c_{2} D_{22}(k, \omega)+m_{3} c_{3} D_{32}(k, \omega) \equiv 0, \\
& m_{1} c_{1} D_{13}(k, \omega)+m_{2} c_{2} D_{23}(k, \omega)+m_{3} c_{3} D_{33}(k, \omega) \equiv 0,
\end{aligned}
$$

where $\{\mathrm{K}, \mathrm{Na}, \mathrm{Cl}\} \leftrightarrow\{1,2,3\}$.

Using the definition for partial ionic conductivities $\sigma_{\alpha}(k, \omega)$,

$$
\sigma_{\alpha}(k, \omega)=q_{\alpha} c_{\alpha} \sum_{\beta} q_{\beta} c_{\beta} D_{\alpha \beta}(k, \omega),
$$


we obtain the expressions:

$$
\begin{aligned}
\sigma_{\mathrm{K}}(k, \omega) & \equiv \sigma_{1}(k, \omega)=c_{1}\left[c_{1} D_{11}(k, \omega)+c_{2} D_{12}(k, \omega)-c_{3} D_{13}(k, \omega)\right], \\
\sigma_{\mathrm{Na}}(k, \omega) & \equiv \sigma_{2}(k, \omega)=c_{2}\left[c_{1} D_{21}(k, \omega)+c_{2} D_{22}(k, \omega)-c_{3} D_{23}(k, \omega)\right], \\
\sigma_{\mathrm{Cl}}(k, \omega) & \equiv \sigma_{3}(k, \omega)=-c_{3}\left[c_{1} D_{31}(k, \omega)+c_{2} D_{32}(k, \omega)-c_{3} D_{33}(k, \omega)\right] .
\end{aligned}
$$

If we multiply each equation in (27) by $m_{1}, m_{2}$ and $\left(-m_{3}\right)$, respectively, add them and use the identities (25), it is easy to obtain the following relation

$$
m_{1} \sigma_{1}(k, \omega)+m_{2} \sigma_{2}(k, \omega)=m_{3} \sigma_{3}(k, \omega),
$$

or

$$
m_{\mathrm{K}} \sigma_{\mathrm{K}}(k, \omega)+m_{\mathrm{Na}} \sigma_{\mathrm{Na}}(k, \omega)=m_{\mathrm{Cl}} \sigma_{\mathrm{Cl}}(k, \omega) .
$$

In the hydrodynamic limit when $(k, \omega) \rightarrow 0$ this result was obtained in [3] by means of the Langevin equation and confirmed directly by molecular dynamics simulations. We note that relation (29) is valid for arbitrary $(k, \omega)$.

Using (28) one can write

$$
m_{\mathrm{A}} \sigma_{\mathrm{A}}(k, \omega)+m_{\mathrm{B}} \sigma_{\mathrm{B}}(k, \omega)=m_{\mathrm{I}} \sigma_{\mathrm{I}}(k, \omega),
$$

for a pseudo-binary electrolyte $\mathrm{AI}-\mathrm{BI}$ with ion charges $q_{\mathrm{A}}=q_{\mathrm{B}}=-q_{\mathrm{I}}$.

\section{Multi-component mixture of charged particles}

Let us now consider the model of a multi-component fluid composed of $N_{\alpha}$ ions with charges $q_{\alpha}$ in the $\alpha$ th species $(\alpha=1,2, \ldots, \nu)$. Mutual diffusion coefficients $D_{\alpha \beta}(k, \omega)$ of the system should satisfy the relations (13). For partial ionic conductivities one has the definition (26), so that if we multiply $\sigma_{\alpha}(k, \omega)$ by $m_{\alpha} / q_{\alpha}$ one can write

$$
\sum_{\alpha} \frac{m_{\alpha}}{q_{\alpha}} \sigma_{\alpha}(k, \omega)=\sum_{\alpha} m_{\alpha} c_{\alpha}\left[\sum_{\beta} q_{\beta} c_{\beta} D_{\alpha \beta}(k, \omega)\right]=\sum_{\beta} q_{\beta} c_{\beta}\left[\sum_{\alpha} m_{\alpha} c_{\alpha} D_{\alpha \beta}(k, \omega)\right] .
$$

Taking into account (13) we finally obtain

$$
\sum_{\alpha} \frac{m_{\alpha}}{q_{\alpha}} \sigma_{\alpha}(k, \omega) \equiv 0
$$

This is a rather general result that includes, as a particular case, the relation (30), derived above. For example, using (32) it is easy to obtain the relation for partial ionic conductivities for pseudobinary four-component mixture $A_{n} I_{l}-B_{k} Y_{j}$ with different charges of ions.

A more complicated case corresponds to the model of a $(\nu+\bar{\nu})$-component fluid that is composed of $N_{\alpha}$ ions with charges $q_{\alpha}(\alpha=1,2, \ldots, \nu)$ and $N_{\bar{\alpha}}$ neutral particles belonging to the $\bar{\alpha}$ th species $(\bar{\alpha}=1,2, \ldots, \bar{\nu})$. In this case the equation (31) should be rewritten in the form

$$
\sum_{\alpha=1}^{\nu} \frac{m_{\alpha}}{q_{\alpha}} \sigma_{\alpha}(k, \omega)=\sum_{\beta=1}^{\nu} q_{\beta} c_{\beta}\left[\sum_{\alpha=1}^{\nu} m_{\alpha} c_{\alpha} D_{\alpha \beta}(k, \omega)\right]=-\sum_{\beta=1}^{\nu} q_{\beta} c_{\beta}\left[\sum_{\bar{\alpha}=1}^{\bar{\nu}} m_{\bar{\alpha}} c_{\bar{\alpha}} D_{\bar{\alpha} \beta}(k, \omega)\right],
$$

where the relations (13) have been used. Note that the index $\bar{\alpha}$ denotes the neutral particles. Therefore, on the right hand side of (33) the effects of mutual diffusion of all the ions in solvent are included. It is obvious that in particular case of a ternary mixture of charged particles in neutral solvent, the expression (24) can be easily recovered from (33). 
The expression (33) can be further simplified if we introduce two new densities, namely the mass density of solvent $\hat{M}_{\mathbf{k}}$ (formed by neutral particles only) and the charge density $\hat{Q}_{\mathbf{k}}$ :

$$
\hat{M}_{\mathbf{k}}=\sum_{\bar{\alpha}=1}^{\bar{\nu}} m_{\bar{\alpha}} c_{\bar{\alpha}} \hat{N}_{\mathbf{k}, \bar{\alpha}}, \quad \hat{Q}_{\mathbf{k}}=\sum_{\alpha=1}^{\nu} q_{\alpha} c_{\alpha} \hat{N}_{\mathbf{k}, \alpha} .
$$

Now we can rewrite (34) in the form

$$
\sum_{\alpha=1}^{\nu} \frac{m_{\alpha}}{q_{\alpha}} \sigma_{\alpha}(k, \omega)=-D_{\mathrm{MQ}}(k, \omega)
$$

where the generalized transport coefficient

$$
D_{\mathrm{MQ}}(k, \omega)=\sum_{\bar{\alpha}=1}^{\bar{\nu}} \sum_{\beta=1}^{\nu} m_{\bar{\alpha}} c_{\bar{\alpha}} D_{\bar{\alpha} \beta}(k, \omega) q_{\beta} c_{\beta}
$$

describes the diffusive ion-solvent cross-correlations. The relation (35) yields, in fact, the most general form of identity valid for partial ionic conductivities of classical systems of charged particles at arbitrary $(k, \omega)$.

\section{Conclusions}

It is shown that the general relations, that link partial ionic conductivities in a binary mixture of charged particles, three- and four-component pseudo-binary molten salts as well as in multicomponent classical fluids of charged particles, can be derived by means of rigorous expressions, obtained previously for the mutual diffusion coefficients of multi-component liquids. Some of these relations generalize the results known in the literature (see, for instance, [2]), but most of them are new. Moreover, all of these relations are valid for $(k, \omega)$-dependent quantities and this, in particular, explains the results of molecular dynamic simulations of the frequency-dependent partial conductivities carried out [10] for molten $\mathrm{NaCl}$ and $\mathrm{NaI}$.

\section{References}

1. Sundheim B.R., J. Phys. Chem., 1956, 60, 1381.

2. Koishi T., Kawase S., Tamaki S., J. Chem. Phys., 2002, 116, 3018; Koishi T., Tamaki S., J. Chem. Phys., 2004, 121, 333.

3. Matsunaga S., Koishi T., Tamaki S., Proc. of the AIP Conference, 2008, 982, 399.

4. Mryglod I., Condens. Matter Phys., 1997, 10, 115.

5. Mryglod I., Bryk T., Kuporov V., Collective dynamics in ionic liquids: A comparative study with nonCoulombic fluids. - In: NATO Science Series II, Eds. D. Henderson, M. Holovko, A. Trokhymchuk, Vol. 206, 2005, p. 109.

6. Kubo R.J., Phys. Soc. Jpn., 1957, 12, 570.

7. Zubarev D.N., Morozov V.G., Röpke G., Statistical mechanics of nonequilibrium processes, Vol. 2: Relazation and hydrodynamic processes. Akademie Verlag, Berlin, 1997.

8. Hansen J.P., McDonald I.R., Phys. Rev. A, 1975, 11, 2111.

9. Trullas J., Padro J.A., J. Chem. Phys., 1993, 99(5), 3983.

10. Koishi T., Tamaki S., J. Phys. Soc. Jpn., 1999, 68, 964. 


\title{
Деякі строгі співвідношення для парціальних провідностей в іонних рідинах
}

\author{
І. Мриглод $\frac{112, \text { В. Купоров }}{2}$ \\ 1 Інститут фізики конденсованих систем НАН України, 79011 Львів, вул. Свєнціцького, 1 \\ 2 Інститут прикладної математики і фундаментальних наук Національного університету “Львівська \\ політехніка", 79013 Львів, Україна
}

\begin{abstract}
Стартуючи з точних співвідношень, що були виведені нами нещодавно для узагальнених коефіцієнтів переносу в багатокомпонентних плинах, ми отримали кілька строгих співвідношень для парціальних провідностей в іонних зарядово-асиметричних сумішах. Для найпростішого випадку зарядовосиметричної бінарної системи таке співвідношення було виявлене експериментально Сундхеймом більш ніж 50 років тому і відоме як "універсальне золоте правило". Розглянуто також деякі більш складні моделі, що описують, зокрема, випадки потрійних та багатокомпонентних сумішей. Виведено загальне співвідношення для парціальних іонних провідностей багатокомпонентного іонного плину. Показано, що такого типу співвідношення $€$ фактично лише одним з прикладів більш широкого класу виразів, що дійсні для $(k, \omega)$-залежних величин.
\end{abstract}

Ключові слова: іонні рідини, коефіцієнти переносу, коефіцієнти взаємної дифузії, іонна провідність, розплави солей 